\title{
Mining Map Interaction Semantics in web-based Geographic Information Systems (WebGIS) for Usability Analysis
}

\author{
René Unrau, Christian Kray \\ r.unrau@uni-muenster.de, c.kray@uni-muenster.de
}

Institute for Geoinformatics, University of Münster, Germany

\begin{abstract}
Due to the increased availability of geospatial data, web-based geographic information systems (WebGIS) have become more popular in recent years. However, the usability of these systems poses new challenges as user interactions are strongly affected by the map and are thus different from interactions with traditional user interface elements. In this paper, we propose a method for evaluating the usability of web-based geographic information systems by analyzing user intentions through map interaction patterns. We use a pattern mining algorithm to extract frequent interaction sequences from user sessions and label these with their interaction semantics that represent the users' immediate intentions. To evaluate our approach, we conducted a user study with 60 participants in a WebGIS scenario and identified varying user strategies for a selection task based on two different geovisualizations. Our results indicate that the chosen approach can uncover the underlying intentions of users' interaction patterns and facilitate insights into the usability of WebGIS.
\end{abstract}

Keywords. template, formatting, reproducible paper guidelines

\section{Introduction}

The increased availability of geospatial data, public participation initiatives, and the technical capabilities of modern web browsers have led to the proliferation of web-based geographic information systems (WebGIS) across multiple platforms (mobile and desktop) in recent years. As a result, the variety of WebGIS contents and applications has grown and includes, for example, extensive map search engines like Google Maps ${ }^{1}$ for contemporary points of interests, fully-featured map builder and exploration tools like the ArcGIS Map Viewer $^{2}$ for businesses and organizations as well as open government initiatives for citizen participation in urban planning processes (Rall et al., 2019). Some of these systems with extensive functionality may require complex user interfaces while, for example, a visualization of search results is only built for simple purposes (Fechner et al., 2015; Lobo et al., 2015; Roth and MacEachren, 2016). Thus, users' knowledge of geospatial visualization and interactions differs greatly and the design of user interfaces (UIs) might yield diverse and potentially conflicting requirement (Roth et al., 2015b; Hoover et al., 2014; Traynor and Williams, 1995). However, all users expect good usability. As a result, designing WebGIS UIs is difficult and requires evaluation approaches that go beyond efficiency and effectiveness to evaluate the usability (Rzeszewski and Kotus, 2019; Poplin et al., 2017; Kiefer et al., 2017).

Evaluating the usability of a WebGIS is difficult, too, as the map represents a central component and is thus the target of most interactions. Compared to traditional UI elements (such as buttons, sliders, and dropdowns) interactions with the map are more strongly affected by its state. For example, the current scale of the map significantly affects users' zoom interactions for a given task. If the zoom level is badly chosen for a specific task users might need longer, are dissatisfied with their experience, or even unable to finish. Treating map interactions like any other functionality can cause problems and misunderstanding as they have a significant impact on how successful users are when accessing a WebGIS and considerably affect which UI elements

\footnotetext{
${ }^{1}$ https://www.google.com/maps/

${ }^{2}$ https://www.arcgis.com/apps/mapviewer/index.html
} 
are used in which order and how often. Consequently, the map state and multi-scale navigation technique affects the efficiency, effectiveness and satisfaction of users. It is, therefore, essential to thoroughly evaluate map interactions to understand these issues better and to assess the usability of WebGIS.

Companies like Google have been doing work on the usability of their web mapping products for more than 10 years (Riegelsberger and Nakhimovsky, 2008). To create interaction models that are independent of a particular interface, research in the field of cartography has evolved taxonomies and topologies that describe single actions such as search, selection, and manipulation (Crampton, 2002; Roth, 2012) in geographic maps. Recording and analyzing such actions allows for optimizations of, for example, the ratio of displayed information or recommendations of individual spatial content (Tahir et al., 2012; Aoidh et al., 2009; Weakliam et al., 2005). Despite this trend, there is only few research that analyzes the semantics of map interaction sequences and thus the usability of the used WebGIS (Roth and MacEachren, 2016; Unrau et al., 2017). Map interaction semantics are the logical aspects of the process that underlies the user's actions and thus reflect the user's immediate intentions. The interpretation of interaction patterns through semantic descriptions may facilitate the understanding of these intentions and reveal further insights into the usability of the application that would otherwise remain hidden.

In this paper, we propose an approach for identifying and analyzing map interaction semantics in geospatial tasks for usability assessment. We (a) instrument WebGIS clients to collect map interactions, (b) apply data mining techniques to retrieve recurring patterns of action sequences from this data, and (c) create labels for the resulting map interaction semantics to (d) analyze the users' behavior for usability assessment. To evaluate our approach, we conducted a user study with 60 participants in a WebGIS scenario. While the overall goal for all participants was the same, we created two different geovisualizations and analyzed differences in the map interaction semantics. In contrast to traditional usability measures, our approach explicitly considers the semantics of map interactions and facilitates the comparison of user strategies in WebGIS workflows beyond efficiency and error rates.

Our contributions are (1) a new approach for evaluating the usability of WebGIS by comparing map interaction semantics through empirically derived interaction patterns; and (2) the usability assessment of an adapted geovisualization method for a selection task based on our approach. Although we only investigate a small set of map interactions (pan, zoom-in/-out, and select), our approach is the first step towards a usability assessment for WebGIS based on map interaction semantics.

The remainder of this paper is structured as follows: First, we review related work in the fields of multi- scale navigation as well as user session analysis and map interaction semantics. Next, we provide the motivation and rationale for our approach and describe the steps that are required to generate map interaction semantics. In section four, we evaluate the approach in a realistic user study that we conducted with 60 participants. The discussion section gives an overview of the implications and limitations of the obtained results and our approach. The paper concludes with a summary of our key findings and contributions.

\section{Related Work}

In this section, we present work that is related to WebGIS evaluation scenarios and software instrumentation in this domain. First, we provide an introduction to multi-scale navigation. Second, we discuss existing evaluation approaches that use map interactions to analyze user behavior. Finally, we show how map interaction semantics have been defined and manually derived in previous work to detect the underlying user intentions.

\subsection{Multi-scale navigation}

Multi-scale navigation is a technique that is used by systems with varying uses of space, time or visual effect to allow users to attain both focused and contextual views of their information space (Cockburn et al., 2009). It was developed to address the breadth of information that often can't be conveniently displayed at one time on a single screen or view. The resulting interfaces allow users to work at multiple levels of detail. Evaluations of multi-scale navigation need to identify effective and ineffective uses of these interfaces. Therefore, terms like the level of details or the visible extent are translated into a computational model, which is used to calculate an analytic solution for optimal animations (Furnas and Bederson, 1995). These models can be tested via user experiments that include free parameters like animation speed. For example, JellyLens is a context-aware and adaptive lense technique that smoothly integrates two levels of details in one view (Pindat et al., 2012). In a controlled experiment the authors showed that this improves the visibility of content in the focus region and preserves a larger part of the context region. Similarly, PolyZoom allows multi scale and multi focus exploration of $2 \mathrm{D}$ spaces by building hierarchies of focus regions, stacked on each other such that each subsequent level shows a higher magnification (Javed et al., 2012). In their validation, the authors showed that this approach performed better then contemporary standard techniques. These evaluations usually include quantitative measures such as the task completion time, number of interactions, and error rate to rate the effectiveness of novel approaches. 
In this paper, we aim to go beyond these numerical results and evaluate the usability of an interface by interpreting the exact interaction patterns of participants and analyzing their sessions.

\subsection{User Session Analysis}

In the past, large-scale analyses of map interaction data have been conducted for various reasons. For example, Aoidh et al. investigated mouse trajectories on the map element to understand the geospatial interests of users (Aoidh et al., 2009). They captured the users' mouse movements, clustered the geographic locations of the mouse cursor positions and visualized the results for exploratory analysis on a new map. Although this approach is sufficient for obtaining insights into the geospatial interests of users, it does not incorporate interactions with traditional UI elements that may provide tools for working with the geospatial data on the map. When a user moves the mouse cursor to a UI element next to the map (e.g., to toggle between various geographic layers) a gap between the following mouse cursor positions is created (Tahir et al., 2012).

Using eye-tracking for data collection is in particular interesting for applications that focus on exploratory usages, such as WebGIS which governmental authorities provide to integrate citizens into planning processes. By tracking the users' focus points and focus duration, eye-tracking has been applied for investigating map interactions (May and Gamble, 2014; Kiefer et al., 2017; Manson et al., 2012) or users' exploration of buttons and icons (Alaçam and Dalcı, 2009; Çöltekin et al., 2009) in WebGIS. However, additional hardware is required and needs to be calibrated for each user.

\subsection{Map Interaction Semantics}

Sequential patterns of map interactions can be extracted for interpreting the users' intentions and provide helpful information. For example, Hiramoto et al. defined operation chunks and complex chunks to query the web for information related to the users' map interactions and visible contents (Hiramoto and Sumiya, 2006). Operation chunks describe "operation sequences that carry meaning" and complex chunks "reflect the users' intentions". A hierarchy of operations (e.g., panning), operation chunks (e.g., narrowing-down) and complex chunks (e.g., comparing points) can then be used to conclude from the users' behavior. Hirose et al. extended this approach and enhanced map interfaces with an additional overview map (Hirose et al., 2007). They also provide a link between the inferred intention and the spatial content by determining the center point or a spatial area for complex chunks. Although their model represents a good baseline for aggregating single map interactions into patterns, the analysis of user intentions for usability evaluation has not been evaluated yet.

Roth developed the idea of interaction primitives for cartographic interfaces by giving an overview of existing taxonomies that focus on the stages of interaction (Roth, 2012). He investigated the usability of a WebGIS by creating relationships between interaction primitives and intentions to articulate user personas in a controlled interaction study (Roth and MacEachren, 2016). In this paper, we take the next logical step beyond prior research, reporting on a large-scale user study to learn how variations of the UI impact users' intentions for WebGIS. In particular, we will answer the question if different map-based UIs have an impact on the users' interaction patterns for a predefined task.

\section{Approach}

The overall goal of our approach is to detect common interaction patterns in WebGIS sessions and provide semantic labels which aim to describe the users' immediate intentions. We thus complement traditional metrics to evaluate the efficiency and effectiveness by explicitly capturing map-related aspects and interactions. To accomplish this task, we design and evaluate a prototypical toolkit for integration into existing WebGIS that combines tailored data collection and pattern mining techniques. The resulting map interaction semantics may facilitate the identification of usability issues and support the improvement of map UIs.

To capture the users' map interactions, we instrument the client software of a WebGIS and send logs with meta information to a central database. Next, we identify common interaction sequences by applying a wellknown pattern mining algorithm to this dataset. Finally, we manually interpret these patterns and assign semantic labels. The first part of this non-intrusive and scalable approach can be automated and is, therefore, implemented into a holistic toolkit that collects the required data and extracts map interaction patterns for analysis. In the following, we provide an overview of each step.

\subsection{Software Instrumentation}

Instrumenting software for usability analysis has become a common technique in HCI research (Lazar et al., 2010). The goal is to collect the required data automatically and facilitate remote user studies. Although the initial development cost might be higher, the instrumented software can be mass deployed and thus reduce the effort for experimenters. Furthermore, instrumenting software allows the conduction of user studies in the user's environment and, thereby, reduces experimenter bias and novelty effects. As modern WebGIS use a variety of web browser technologies to 
provide tools for displaying, editing and analyzing geospatial data, many user interactions cannot be retrieved from server logs but must be collected from within the client (i.e., the users' web browser). To implement our approach for the evaluation, we make use of three software components.

First, we instrument the JavaScript source code of the WebGIS client by connecting user interaction events with a custom logging script. Most WebGIS rely on mapping frameworks to provide geographic maps and tools in web browsers (Roth et al., 2015a). Frequently, these frameworks already provide programming interfaces that allow developers to subscribe to user interaction events and can thus be used for our purpose. For our evaluation, we connected our logging script to the ArcGIS API for JavaScript ${ }^{3}$ and subscribed to the following events which may be carried out via various input types (e.g., mouse click, keyboard, touch gestures):

- Zoom In (I) Increasing the scale of the map to reveal more details.

- Zoom Out (O) Decreasing the scale of the map to get an overview.

- Pan (P) Moving the visible extent without changing the scale to explore the map content.

- Select (S) Selecting a map entry to reveal more details about it or adding it to a collection

However, our implementation is framework agnostic and accepts any events that are configured on startup. Listing 1 shows a JavaScript code snippet that demonstrates how events of a mapping framework are connected to our logging module. This lightweight approach allows developers to easily instrument the code of other WebGIS clients as they only need to learn the interfaces for retrieving the desired information. While this process still requires access to the WebGIS source code the required effort is low. The developer does not need profound programming skills or an extensive knowledge of the WebGIS code base for carrying out this task. For our evaluation, the instrumentation of the JavaScript code for an existing WebGIS client was completed within a few hours.

Listing 1. JavaScript sample code for connecting logging module to third-party mapping framework events

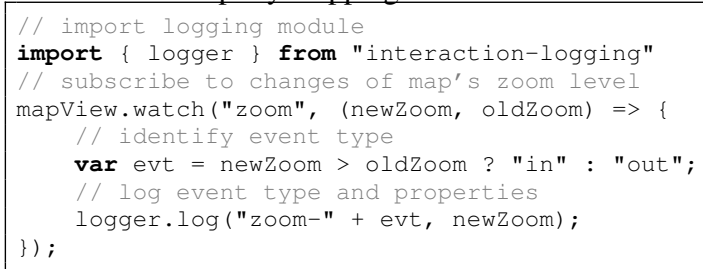

${ }^{3} \mathrm{https}: / /$ developers.arcgis.com/javascript/latest/
In our logging module we add additional metadata, such as the timestamp, to the event data and send the information to our second software component.

We used the open-source database engine Elasticsearch $^{4}$ as a central archive that runs on a central server. Elasticsearch provides a schema-less index and can thus be filled with custom data fields without adjusting the data model. These capabilities ensure that our approach is customizable and facilitates the realization of further logging scenarios in the future with little effort.

Finally, in our third software component, we extract patterns in the logged data via a python script that automatically retrieves the map interaction data from our Elasticsearch instance and extends an implementation of GSP (Prado Lima, 2019) to mine the recorded user sessions for reoccurring patterns.

\subsection{Sequential Pattern Mining}

WebGIS capabilities are usually limited compared to rich desktop GIS because WebGIS are accessed via web browsers and sometimes mobile devices with less computing power. For example, WebGIS do not provide extensive functionality for creating custom maps and analyzing large datasets in the client but instead consume preprocessed data and provide carefully selected tools. Therefore, most WebGIS are built with focused scenarios in mind and designers strive to identify the most usable interface for the task at hand. Nevertheless, these scenarios may consist of multiple steps that result in numerous (map) interactions and are thus difficult to interpret. The extraction of recurring interaction patterns facilitates the identification of typical user behavior which, then, must be analyzed.

We will make use of Sequential Pattern Mining for this extraction process. Sequential pattern mining is an area of Data Mining or Knowledge Discovery that scans and detects all data sequences which contain recurring patterns (Srikant and Agrawal, 1996). Developed initially to analyze customer behavior, it also serves our purpose to interpret the underlying intentions of WebGIS interactions. We adapt GSP (Generalized Sequential Pattern), an improved pattern detection algorithm that scales linearly with the number of data sequences (Agrawal and Srikant, 1995). This level-based algorithm iterates multiple times through the dataset. First, the frequency (support of a pattern) of all sequences with the length of one $(n=1)$ is determined. Next, non-frequent sequences are removed and the remaining sequences are used for the next iteration that determines the frequency of patterns with the length of two $(n=2)$. This procedure is repeated until the set of frequent items is empty for the next iteration.

\footnotetext{
${ }^{4}$ https://www.elastic.co/elasticsearch/
} 
A typical uses case of the GSP algorithm is the identification of shopping patterns in a web shop to infer what customers might buy next. The input sequences, in this case, are the ordered lists of previously bought items for each customer. The resulting patterns are frequent transaction sequences that could be used to display recommendations to customers. Customers who buy some other articles in between also support a pattern that does not include every of their items. Note that these transactions do not need to be consecutive. For our application of the algorithm, we use lists of interactions that represent WebGIS user sessions as input sequences.

A minimal support threshold (minsup) must be defined as an additional parameter $[0,1]$ and determines the number of occurrences of sequences in the dataset to remain for the next iteration. The algorithm, then, returns the sequences for all iterations that fulfill this criteria and can thus be recognized as common patterns in the dataset. The minsup value is usually determined by trial and error until a value is found that reveals interesting patterns. However, the size of the dataset $(x)$ plays an important role for the minsup value. If the dataset is small, the threshold must be set to a high value while a low value is required for large dataset. The exponential decay function (Fournier-Viger, 2010) can be used to describe a curve that represents such a behavior:

$$
\operatorname{minsup}(x)=\left(e^{-a x-b}\right)+c
$$

The parameters $a, b$, and $c$ are static values that must be adjusted to make the curve behave differently. The value of $c$ represents the minimal minsup value (i.e. the threshold for large datasets) of the curve. The values of $a$ and $b$ determine the decline of the curve (i.e. the minsup values will be lower for larger datasets) and thus describe how long it will take before the minsup value converges to the value of $c$.

\subsection{Semantic Labeling}

Finally, we assign descriptive labels to the detected interaction patterns by interpreting the semantics. We distinguish between basic actions, the fundamental operations for manipulating digital maps (i.e. the recorded input events), and complex actions that are sequences of basic actions and represent more complex interaction semantics.

Complex actions are the result of our sequential pattern mining algorithm. These actions facilitate the interpretation of user intentions that are executed via multiple basic actions and, thus, reflect broader user strategies for the overall task (e.g., Relative Position Confirmation). In the following, we will denote the length of interaction patterns that underlie our complex actions as complexity. Complex actions will help us to detect if there is common user behavior, depending on the chosen threshold for recognizing patterns and the frequency of identified patterns.

\section{Evaluation}

To evaluate our approach, we conducted a user study and extracted interaction patterns for a localization tasks. Two groups of participants were provided with different multi-scale navigation techniques. The first group was equipped with a novel technique that made use off-screen indicators (OSI) (Gustafson et al., 2008; Ballatore, 2019). These OSI consists of triangles whose sides can be traced and extrapolated to locate the off-screen object (i.e. map features that are outside the currently visible view) (Baudisch and Rosenholtz, 2003). By tracking the relative position of these objects continuously users are able to estimate where the triangle's sides intersect and navigate precisely to the desired entry (Figure 1). Additionally, an alphabetical coding could be used by this group to match the features on the map to the corresponding row in the table below. The coding was displayed next to the OSI on the map and as an attribute in the table rows to facilitate the assignment of both representations. The second group used a traditional geovisualization technique for dynamic maps that simply highlights the map features with a point symbol and does not indicate if and where additional features might be located outside of the current map view. This group also had to mentally match the table rows to the features on the map by either hovering the one of them which highlighted the correspondent feature or row (Figure 2). We randomly assigned participants to one of the two groups in a between-group user study.

Participants: We opportunistically recruited 60 participants during a user conference of the tested WebGIS to participate in the study using our optimized OSI version. The primary criteria for participation in our study included basic knowledge and experience with WebGIS (i.e., participants were actual end-users). The resulting sample set of participants consisted of regular users with high levels of motivation and WebGIS expertise. Our sample size of $n=60$ for two different scenarios is sufficient to achieve a high level of thoroughness based on recommendations for usability testing (Nielsen and Landauer, 1993).

Material and Procedure: We conducted the study during the mentioned conference in a quiet area at the conference venue. As the data collection was performed automatically, our simple usability setup consisted of a laptop computer, an external monitor, and a mouse that were used by the participants during testing. We completed all sessions over two days, with our setup remaining in the same configuration throughout the period. 


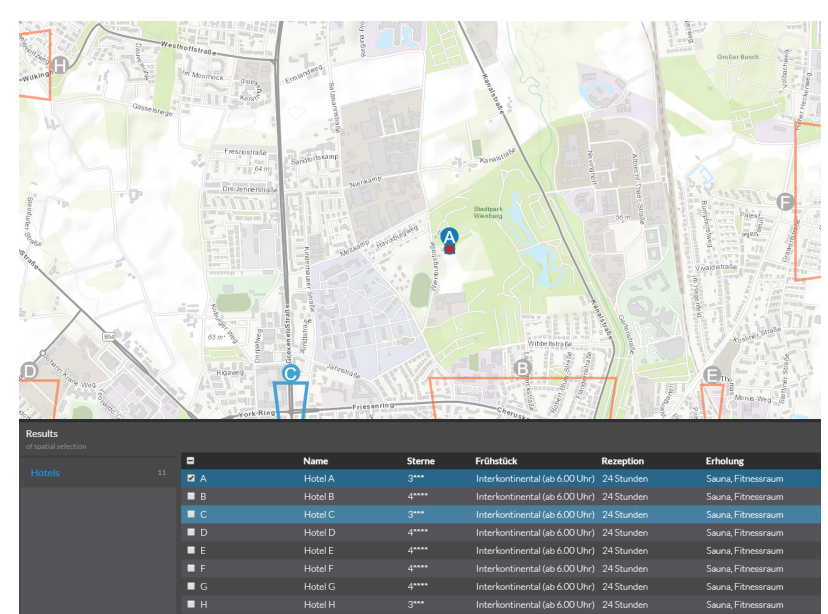

Figure 1. The first group was presented with off-screen indicators that reveal map entries which are not visible in the current viewport of the screen. Our optimized visualization extends the indicators with an alphabetical coding to simplify the assignment of individual values from a table.

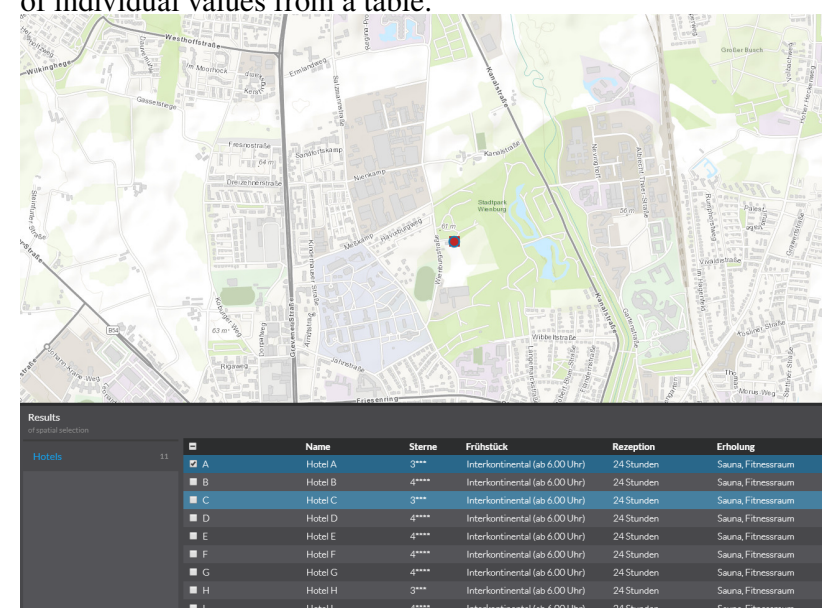

Figure 2. The second group was equipped with a common geovisualization that display map entries as simple dots on the map. The connection between map and table entries is not initially available, and users must hover or select table entries to mentally establish the connection.

Similar to the idea of a usability kiosk (Nielsen, 1993), we invited passing-by persons to participate in a 10minute user study that investigated an experimental design for visualizing the relationship between data in a table and on the map. First, participants sat down in front of our setup that guided users through the required steps and automatically assigned them to one of our two groups. Next, we asked participants to rate their experience with GIS on a Likert scale based on the following statement: I have experience in working with GIS (1: strongly disagree, 5: strongly agree). Before starting with the study, participants also had to confirm an informed consent form about the anonymously collected data. Without any initial exploration period, every participant was tasked to select property entries on the map that had a rectangular shape and an area bigger than a $5 \mathrm{~km}^{2}$. Attributes of the map entries, such as the area, were displayed in the table below the map. By using the checkbox in one of the table rows, the corresponding entry was added to or removed from the set of selected entries. This task layout was created to require participants to interact with the map as well as with the table. We chose the initial extent of the map view not to show all entries and, thus, required participants to interact with the map element via pan or zoom actions. During the study, participants were allowed to ask questions for clarification, and all sessions were finished with a set of properties that fulfilled the given criteria.

\section{Results}

In total, we collected 1248 map interactions from 60 user sessions (44 male, 16 female). Both geovisualization types were used by two groups of 30 participants each and an overall mean age of $38.3(\sigma=10.13)$ and a self-rated GIS experience of 4.6 ( $\sigma=0.95$, Likert scale: 1 to 5). An independent-samples t-test was conducted to compare the task completion times. There was no significant differences in the times with OSI $(\mathrm{M}=89.3$ seconds, $\mathrm{SD}=41.7)$ and without OSI $(\mathrm{M}=$ 92.2, $\mathrm{SD}=86$ ) conditions; $\mathrm{t}(54)=0.2658, \mathrm{p}=.7914$. About $60 \%$ of the users in the scenario with OSI selected all property entries that fulfilled the required criteria. In the scenario without OSI, the success rate was $73 \%$. In both scenarios, properties that did not fulfill the required criteria were selected by 6 or less participants. We performed a chi-square test of independence to examine the relation between the geovisualization and the count of basic actions. The relation between these variables was significant, $\mathrm{X} 2(3, \mathrm{~N}=60)=7.94$, $\mathrm{p}=.47$. Users with OSI were more likely to perform basic actions.

For mining complex interaction in our dataset, we determined a threshold of $30 \%$, meaning that at least $30 \%$ of the groups' participants (i.e., user sessions) must have performed a particular sequence to be recognized as a pattern. This minsup value was established via the exponential decay function (final parameters: $x=1248 a=-0.0017, b=-0.2$, and $c=0.2$ ). The pattern mining algorithm detected 11 complex actions which were interpreted through the following map interaction semantics:

- Detail Verification (I-O): Zooming in to inspect details of the entry or its surroundings before zooming out again to establish the previous scale.

- Detail Selection (I-S): Zooming in to inspect details and select an entry.

- Inspection (I-P): Zooming in and panning the map to investigate details in a particular area. 


\begin{tabular}{l|c|c|c|c|l}
\multirow{2}{*}{ Pattern } & \multicolumn{2}{|c|}{ w/ indicators } & \multicolumn{2}{c|}{ w/o indicators } & Semantic Description \\
& Total & Users & Total & Users & \\
\hline P, S & 46 & 22 & 28 & 15 & Browse Selection \\
P, I & 21 & 15 & - & - & Narrow-Down Search \\
P, O & 20 & 14 & 14 & 11 & Spread-Out Search \\
I, S & 19 & 16 & - & - & Detail Selection \\
I, P & 19 & 12 & - & - & Inspection \\
I, O & - & - & 17 & 10 & Detail Verification \\
O, P & 30 & 15 & 22 & 14 & Spread-Out Search \\
O, I & 18 & 15 & - & - & Rel. Pos. Confirmation \\
\hline I, S, P & 9 & 9 & - & - & Scale Choice \\
S, O, I & 9 & 9 & - & - & Surroundings Inspection \\
O, P, S & 11 & 9 & 13 & 12 & Spread-Out Selection \\
S, P, S & 29 & 15 & - & - & Supervised Selection \\
P, S, P & 31 & 16 & - & - & Supervised Selection \\
\hline P, S, P , S & 18 & 9 & - & - & Supervised Selection \\
S, P , S , P & 23 & 13 & - & - & Supervised Selection
\end{tabular}

Table 1. Complex actions with their total and user count for scenario with indicators and without indicators (both were conducted by 30 participants each). Complex actions that did not pass the threshold for the group (30\% of users) were ignored here. - I: Zoom In; O: Zoom out; P: Pan; S: Select

With Off-Screen Indicators

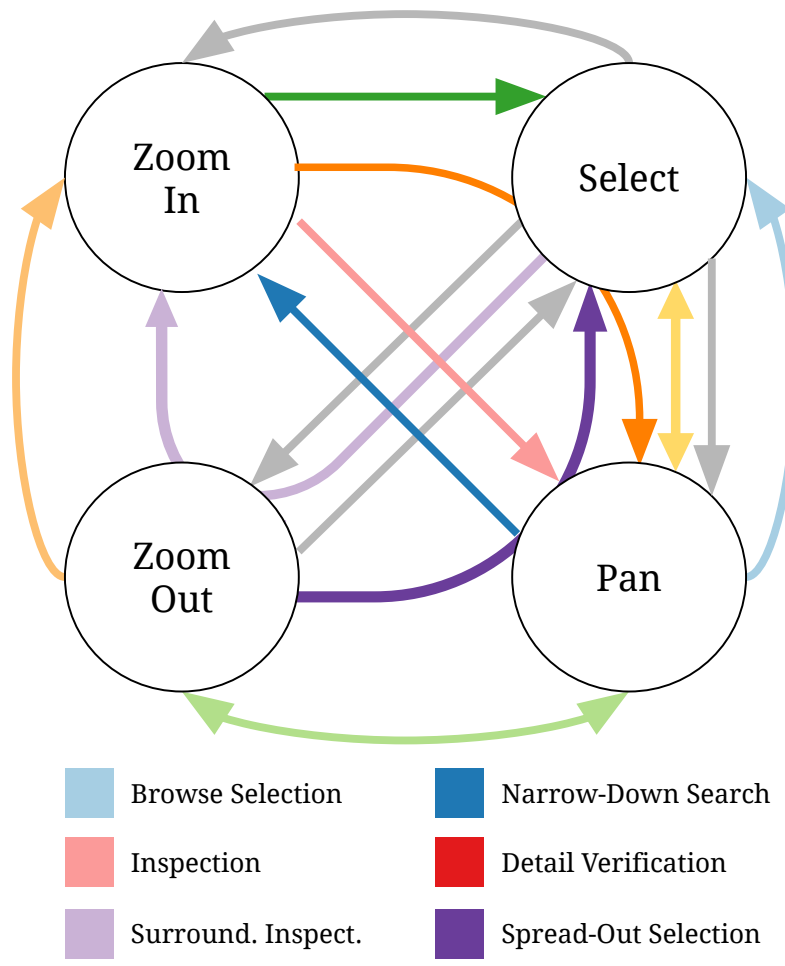

Without Off-Screen Indicators

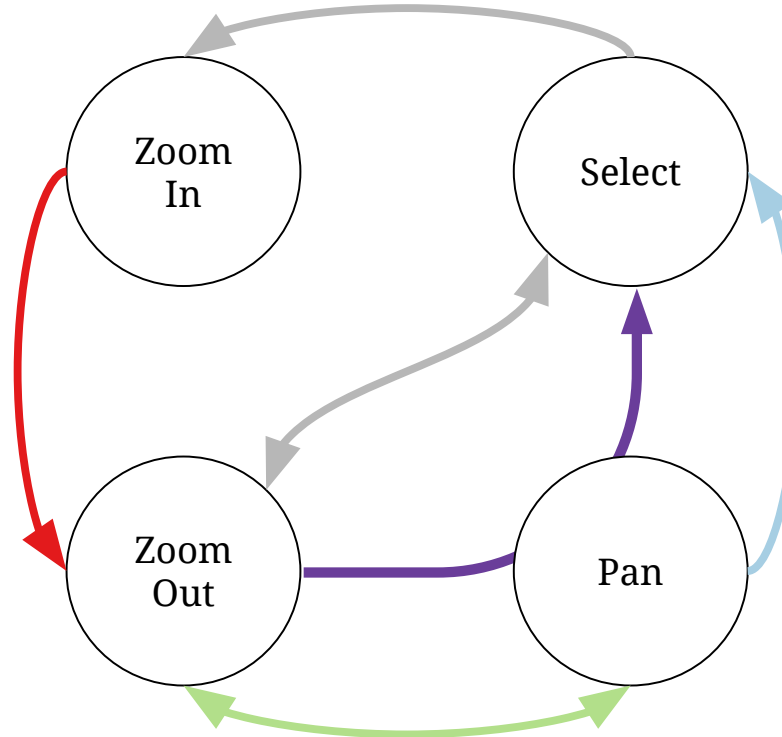

Spread-Out Search

Rel. Pos. Confirm.

Supervised Selection
Detail Selection

Scale Choice

No Semantic

Figure 3. Complex actions and their semantic descriptions which passed the defined threshold (30\% of user sessions with one or more executions). 
- Relative Position Confirmation (O-I): Zooming out to localize the current position based on the surroundings before zooming in again to establish the previous scale.

- Spread-Out Search (P-O or O-P): Searching for further entries by increasing the visible area through panning the map and zooming out to move on to a different location (faster).

- Narrow-Down Search (P-I): Searching the map by panning around before zooming in to a particular area.

- Browse Selection (P-S): Scanning entries by panning the map before selecting an entry.

- Scale Choice (I-S-P): Reducing the scale to select an entry and panning the map to search for the next entry of interest. As the user keeps the reduced scale after the selection, this semantic also determines a selection of a new scale by the user for his search process.

- Surroundings Inspection (S-O-I): Confirming the own position after selecting an entry to get an overview of remaining entries and their relative position.

- Spread-Out Selection (O-P-S): Zooming out to move to a different selection faster and selects an item here.

- Supervised Selection ([S-]P-S[-P]): Keeping the same scale and continuously selecting entries by panning the map to get to items outside of the visible extent. This semantic manifests in multiple similar interaction patterns that are either subsets or vary in the starting interaction before alternating between Pan and Select.

In contrast to the basic interactions, the side-by-side comparison of these complex actions reveals significant differences between participants in the group with OSI and those without OSI (see Figure 3). While we found ten different semantic interaction patterns in the group of participants with OSI, participants without OSI executed only four different patterns (see Table 1). As the total interaction count is similar for both groups, the small set of map interaction semantics for the group without OSI (as well as the often lower execution count for the remaining semantics) indicates a lack of a common strategy for these participants. Instead, we expect a variety of individual patterns below our chosen threshold for this group.

Browse Selection represents the most often detected map interaction semantic for both groups (see Table 1) and could describe the participants' aim to scan the map with the same scale while selecting entries. However, participants that were provided with OSI show a higher proportion for this semantic.

\begin{tabular}{l|c|c|c|c}
\multirow{2}{*}{ Pattern } & \multicolumn{2}{|c|}{ w/ indicators } & \multicolumn{2}{c}{ w/o indicators } \\
& Total & Users & Total & Users \\
\hline S, P & 46 & 20 & - & - \\
S, I & 12 & 10 & 16 & 13 \\
S, O & 20 & 14 & 23 & 16 \\
O, S & 11 & 10 & 26 & 17
\end{tabular}

Table 2. Frequencies of detected interaction patterns which could not be interpreted and assigned to a map interaction semantic. - I: Zoom In; O: Zoom out; P: Pan; S: Select

Despite the variety of map interaction semantics for the group with OSI, Supervised Selection stands out in our data. This complex action is the result of multiple and similar underlying interaction patterns while also being the only one with a complexity higher than two and an average execution per user above one $(\mathrm{P}, \mathrm{S}, \mathrm{P}: 31 \mathrm{ex}-$ ecutions for 30 participants). Thus, the simple alternation of Pan and Select seems to be a characteristic behavior of participants for this scenario. It seems plausible that Supervised Selection is a result of the optimized indicators which direct users to map entries outside of the visible map extent without having to zoom out.

The algorithm detected four additional complex actions, but we could not assign any meaningful map interaction semantic (see Table 2). However, these patterns seem to be exceptions that can be explained in the context they were executed. The S, P pattern is a subset of the more complex Supervised Selection and did not pass the threshold for the group without OSI, for the described reasons. The remaining three patterns without a semantic description could indicate that users carried out their goal to select an entry and begin with a new semantic interaction.

\section{Discussion}

To provide a positive experience for users of a WebGIS, it is essential to assess the usability of these systems. Prior work has analyzed the usage of WebGIS to learn about users' interaction with geospatial data and inform the design of UIs. Roth, for example, compared the performance and used map operators via interaction logs to identify user personas (Roth and MacEachren, 2016). Our findings confirm that map interactions can be used to interpret the users' immediate intentions which are essential for detecting flaws in the UI. This study, therefore, indicates that the steps for retrieving the required data could be carried out by a holistic toolkit that supports UI designers in the decision-making process.

By applying our new approach, presented in this paper, and comparing map interaction semantics through empirically derived interaction patterns we were able to identify that the majority of participants equipped with OSI approached the given localization task with 
the same interaction strategy. By making use of Supervised Selections these users could stay on a map scale that allowed them to verify details on the map that were required for the given task while also being able to pan to the next map entry. In contrast, traditional usability measures, like task completion times, could not indicate significant differences between both groups. We can attribute this to the novelty of OSI for most users that might not result in increased performance initially but requires some learning first. However, our approach can help usability experts to gain deeper insights into user strategies from remotely logged map interaction data and provide indicators that show if UI changes result in changed user behavior.

Our results also indicate open usability issues with the WebGIS that was evaluated. While OSI users were able to make use of the provided indicators the extracted patterns reveal that some of these users performed Relative Position Confirmations. While OSI should support users in navigating to map entries without having to zoom out it seems like these participants still wanted to keep track of their position on the map. Thus adding an overview map to our UI could enhance the usability of the evaluated WebGIS.

In addition, we found that some OSI participants (9 out of 30) performed exactly one Scale choice in their session. This could be an indicator for a poorly chosen initial scale that requires users to zoom in at the beginning of their session before they can benefit from the OSI and focus on the task at hand. Usability designers can use findings like this one to further analyze the logged interaction data to confirm this hypothesis and adjust the initial scale of the evaluated WebGIS.

Although zooming and panning represent the most critical map interactions in WebGIS scenarios, our evaluation covered only a small subset of possible map interactions and a single selection task. Frequently, modern WebGIS provide tools for spatial selections or drawing notes which may lead to more complex interaction patterns. However, as WebGIS are designed to cover only a limited number of workflows, the interpretation of these patterns should be feasible if the aim of the user is known to the analyst.

Regarding our approach, further work is needed to evaluate the correctness of our map interaction semantics. In this study, the assumptions about the underlying intentions were not validated by, for example, conducting follow-up interviews with the participants. While the quantitative results of our study indicate that many users with OSI followed a common strategy, it remains unclear if, for example, the intention of $I$, $O$ was always Detail Verification. However, the design of our focused task, as well as the detected patterns, do not leave room for arbitrary interpretations in this context. Future work should investigate more complex scenarios and validate the resulting map interaction semantics by consulting the study's participants.
Further limitations of our evaluation are the type of our study participants as well as the complexity of the tested geovisualizations. The overall self-rated GIS experience of our users was very high and the chosen approach for recruiting participants on a conference certainly contributes to a homogeneous cohort. Last, the tested scenarios represent simple tasks compared to modern WebGIS that offer numerous tools and data sources. However, our work can be considered a first step in to the investigation of the benefits of interaction semantics for this domain. Instrumenting WebGIS for automatic data collection and pattern extraction also simplifies the conduction of future studies in more complex environments and with heterogeneous users.

Furthermore, it would be interesting to investigate the temporal and spatial correlation of interaction patterns within user sessions. Finding out whether users with the same immediate intentions carried out the corresponding interaction patterns at the same geographic location on the map or at the same point in time relative to their session start could reveal further insights and help to differentiate groups of users with the same strategy further. Therefore, future work could visualize this data as a map layer that can be explored by usability analysts to detect spatial and temporal patterns.

\section{Conclusion}

The evaluation of map interaction patterns is critical for usability assessment in web-based geographic information systems because they differ from interactions with traditional UI elements. Recent trends, such as the Internet of Things, increase the dissemination of geospatial data and the need for appropriate mediums to display and manipulate location information in their context. Dynamic maps as a medium can handle these vast amounts of data and are increasingly accessed by non-expert users via the World Wide Web.

In this paper, we present an approach and toolkit for detecting, describing and comparing map interactions in WebGIS sessions for usability analysis. Map interaction semantics describe the users' immediate intentions while interacting with the WebGIS and, thus, increase insights into user behavior compared to analyzing isolated interactions. To extract these intentions from users' sessions, we mined all user interactions for common patterns as they usually manifest in sequences of interactions. Because the data was collected automatically via an instrumented WebGIS software, our approach facilitates the extraction of map interaction patterns without an experimenter on site or additional hardware, such as eye-tracking devices.

We conducted a kiosk study to investigate the feasibility and potential of our approach. Overall, the result provides evidence that the chosen geovisualiza- 
tion affects the users' immediate intentions for the same task. We showed that off-screen indicators encourage specific user approaches and result in common user strategies, whereas the lack of these indicators results in a variety of individual approaches. Our method, therefore, can be used to compare the influence of UI changes on the users' intentions and assist decision makers in usability evaluations. Our key contributions are a new approach for evaluating the usability of web-based geographic information systems by comparing map interaction semantics through empirically derived interaction patterns; and the usability assessment of an adapted geovisualization method for a selection task based on our approach. In the future, we will extend our toolkit to visualize the results and thus provide more spatial and temporal context to analysts, and we will assess the usefulness of our approach for more complex scenarios.

\section{Data and Software Availability}

The computational environment was provided by using a virtual machine (OS: Windows 10) with an Elasticsearch cluster (version 2.1). For collecting user interactions during our study and retrieving this data for the analysis, the virtual machines were accessed via a local network. As the used open source version of Elasticsearch does not provide any security layer, it is not possible to share any connection details to our datasets. To mine the retrieved data for pattern a publicly available Python implementation of GSP was used (https://doi.org/10.5281/zenodo.3333988).

\section{References}

Agrawal, R. and Srikant, R.: Mining Sequential Patterns, in: Proceedings of the Eleventh International Conference on Data Engineering, ICDE '95, pp. 3-14, IEEE Computer Society, Washington, DC, USA, http://dl.acm.org/citation.cfm? id $=645480.655281,1995$.

Alaçam, O. and Dalcı, M.: A Usability Study of WebMaps with Eye Tracking Tool: The Effects of Iconic Representation of Information, in: Proceedings of the 13th International Conference on Human-Computer Interaction. Part I: New Trends, pp. 12-21, Springer-Verlag, Berlin, Heidelberg, https://doi.org/10.1007/978-3-642-02574-7_2, 2009.

Aoidh, E. M., Wilson, D. C., and Bertolotto, M.: A Study of Spatial Interaction Behaviour for Improved Delivery of Web-Based Maps, in: Proceedings of the 9th International Symposium on Web and Wireless Geographical Information Systems, W2GIS '09, pp. 120-134, SpringerVerlag, Berlin, Heidelberg, https://doi.org/10.1007/978-3642-10601-9_9, 2009.

Ballatore, A.: A Context Frame for Interactive Maps, in: Geospatial Technologies for Local and Regional Development : short papers, posters and poster abstracts of the 22nd AGILE Conference on Geographic Information Sci- ence. Cyprus University of Technology, 17-20 June 2019, pp. $1-5,2019$.

Baudisch, P. and Rosenholtz, R.: Halo: A Technique for Visualizing Off-screen Objects, in: Proceedings of the SIGCHI Conference on Human Factors in Computing Systems, CHI '03, pp. 481-488, ACM, New York, NY, USA, https://doi.org/10.1145/642611.642695, 2003.

Cockburn, A., Karlson, A., and Bederson, B. B.: A Review of Overview+detail, Zooming, and Focus+context Interfaces, ACM Comput. Surv., 41, https://doi.org/10.1145/1456650.1456652, 2009.

Çöltekin, A., Heil, B., Garlandini, S., and Fabrikant, S. I.: Evaluating the Effectiveness of Interactive Map Interface Designs: A Case Study Integrating Usability Metrics with Eye-Movement Analysis, Cartography and Geographic Information Science, 36, 5-17, https://doi.org/10.1559/152304009787340197, 2009.

Crampton, J. W.: Interactivity Types in Geographic Visualization, Cartography and Geographic Information Science, 29, 85-98, https://doi.org/10.1559/152304002782053314, 2002.

Fechner, T., Wilhelm, D., and Kray, C.: Ethermap: Real-time Collaborative Map Editing, in: Proceedings of the 33rd Annual ACM Conference on Human Factors in Computing Systems, CHI '15, pp. 3583-3592, ACM, New York, NY, USA, https://doi.org/10.1145/2702123.2702536, 2015.

Fournier-Viger, P.: Un modèle hybride pour le support à l'apprentissage dans les domaines procéduraux et mal définis, Ph.D. thesis, Université du Québec à Montréal, 2010.

Furnas, G. W. and Bederson, B. B.: Space-Scale Diagrams: Understanding Multiscale Interfaces, in: Proceedings of the SIGCHI Conference on Human Factors in Computing Systems, CHI '95, p. 234-241, ACM Press/Addison-Wesley Publishing Co., USA, https://doi.org/10.1145/223904.223934, 1995.

Gustafson, S., Baudisch, P., Gutwin, C., and Irani, P.: Wedge: Clutter-free Visualization of Off-screen Locations, in: Proceedings of the SIGCHI Conference on Human Factors in Computing Systems, CHI '08, pp. 787-796, ACM, New York, NY, USA, https://doi.org/10.1145/1357054.1357179, 2008.

Hiramoto, R. and Sumiya, K.: Web Information Retrieval Based on User Operation on Digital Maps, in: Proceedings of the 14th Annual ACM International Symposium on Advances in Geographic Information Systems, GIS '06, pp. 99-106, ACM, New York, NY, USA, https://doi.org/10.1145/1183471.1183489, 2006.

Hirose, M., Hiramoto, R., and Sumiya, K.: GeminiMap - Geographical Enhanced Map Interface for Navigation on the Internet, in: Proceedings of the 7th International Conference on Web and Wireless Geographical Information Systems, W2GIS'07, pp. 279-292, Springer-Verlag, Berlin, Heidelberg, 2007.

Hoover, J. H., Sutton, P. C., Anderson, S. J., and Keller, A. C.: Designing and evaluating a groundwater quality Internet GIS, Applied Geography, 53, 55-65, https://doi.org/10.1016/j.apgeog.2014.06.005, 2014.

Javed, W., Ghani, S., and Elmqvist, N.: Polyzoom: Multiscale and Multifocus Exploration in 2d Visual Spaces, 
in: Proceedings of the SIGCHI Conference on $\mathrm{Hu}$ man Factors in Computing Systems, CHI '12, Association for Computing Machinery, New York, NY, USA, https://doi.org/10.1145/2207676.2207716, 2012.

Kiefer, P., Giannopoulos, I., Athanasios Anagnostopoulos, V., Schöning, J., and Raubal, M.: Controllability Matters: The User Experience of Adaptive Maps, Geoinformatica, 21, 619-641, https://doi.org/10.1007/s10707-016-0282-x, 2017.

Lazar, J., Feng, J. H., and Hochheiser, H.: Research Methods in Human-Computer Interaction, Wiley Publishing, Chichester, UK, 2010.

Lobo, M.-J., Pietriga, E., and Appert, C.: An Evaluation of Interactive Map Comparison Techniques, in: Proceedings of the 33rd Annual ACM Conference on Human Factors in Computing Systems, CHI '15, pp. 3573-3582, ACM, New York, NY, USA, https://doi.org/10.1145/2702123.2702130, 2015.

Manson, S. M., Kne, L., Dyke, K. R., Shannon, J., and Eria, S.: Using Eye-tracking and Mouse Metrics to Test Usability of Web Mapping Navigation, Cartography and Geographic Information Science, 39, 48-60, https://doi.org/10.1559/1523040639148, 2012.

May, J. and Gamble, T.: Collocating Interface Objects: Zooming into Maps, in: Proceedings of the 32Nd Annual ACM Conference on Human Factors in Computing Systems, CHI '14, pp. 2085-2094, ACM, New York, NY, USA, https://doi.org/10.1145/2556288.2557279, 2014.

Nielsen, J.: Usability Engineering, Morgan Kaufmann Publishers Inc., San Francisco, CA, USA, 1993.

Nielsen, J. and Landauer, T. K.: A Mathematical Model of the Finding of Usability Problems, in: Proceedings of the INTERACT ' 93 and CHI '93 Conference on Human Factors in Computing Systems, CHI '93, pp. 206-213, ACM, New York, NY, USA, https://doi.org/10.1145/169059.169166, 1993.

Pindat, C., Pietriga, E., Chapuis, O., and Puech, C.: JellyLens: Content-Aware Adaptive Lenses, in: Proceedings of the 25th Annual ACM Symposium on User Interface Software and Technology, UIST '12, p. 261-270, Association for Computing Machinery, New York, NY, USA, https://doi.org/10.1145/2380116.2380150, 2012.

Poplin, A., Guan, W., and Lewis, B.: Online Survey of Heterogeneous Users and Their Usage of the Interactive Mapping Platform WorldMap, The Cartographic Journal, 54, 214-232, https://doi.org/10.1080/00087041.2016.1229248, 2017.

Prado Lima, J. A. d.: GSP-Py - Generalized Sequence Pattern algorithm in Python, https://doi.org/10.5281/zenodo.3333988, 2019.

Rall, E., Hansen, R., and Pauleit, S.: The added value of public participation GIS (PPGIS) for urban green infrastructure planning, Urban Forestry Urban Greening, 40, 264-274, https://doi.org/https://doi.org/10.1016/j.ufug.2018.06.016, 2019.

Riegelsberger, J. and Nakhimovsky, Y.: Seeing the Bigger Picture: A Multi-Method Field Trial of Google Maps for Mobile, in: CHI '08 Extended Abstracts on Human Factors in Computing Systems, CHI EA '08, p. 2221-2228, As- sociation for Computing Machinery, New York, NY, USA, https://doi.org/10.1145/1358628.1358655, 2008.

Roth, R., Donohue, R., Sack, C., Wallace, T., and Buckingham, T.: A Process for Keeping Pace with Evolving Web Mapping Technologies, Cartographic Perspectives, 0, 25-52, 2015a.

Roth, R. E.: Cartographic Interaction Primitives: Framework and Synthesis, The Cartographic Journal, 49, 376-395, https://doi.org/10.1179/1743277412Y.0000000019, 2012.

Roth, R. E. and MacEachren, A. M.: Geovisual analytics and the science of interaction: an empirical interaction study, Cartography and Geographic Information Science, 43, 30-54, https://doi.org/10.1080/15230406.2015.1021714, 2016.

Roth, R. E., Ross, K. S., and MacEachren, A. M.: UserCentered Design for Interactive Maps: A Case Study in Crime Analysis, ISPRS International Journal of GeoInformation, 4, 262-301, http://www.mdpi.com/2220-9964/ 4/1/262, 2015b.

Rzeszewski, M. and Kotus, J.: Usability and usefulness of internet mapping platforms in participatory spatial planning, Applied Geography, 103, 56-69, https://doi.org/https://doi.org/10.1016/j.apgeog.2019.01.001, 2019.

Srikant, R. and Agrawal, R.: Mining Sequential Patterns: Generalizations and Performance Improvements, in: Proceedings of the 5th International Conference on Extending Database Technology: Advances in Database Technology, EDBT '96, pp. 3-17, Springer-Verlag, London, UK, UK, http://dl.acm.org/citation.cfm?id=645337.650382, 1996.

Tahir, A., McArdle, G., and Bertolotto, M.: Identifying specific spatial tasks through clustering and geovisual analysis, in: 2012 20th International Conference on Geoinformatics, pp. 1-6, IEEE Computer Society, Washington, DC, USA, https://doi.org/10.1109/Geoinformatics.2012.6270301, 2012.

Traynor, C. and Williams, M. G.: Why Are Geographic Information Systems Hard to Use?, in: Conference Companion on Human Factors in Computing Systems, CHI '95, pp. 288-289, ACM, New York, NY, USA, https://doi.org/10.1145/223355.223678, 1995.

Unrau, R., Ostkamp, M., and Kray, C.: An Approach for Harvesting, Visualizing, and Analyzing WebGIS Sessions to Identify Usability Issues, in: Proceedings of the ACM SIGCHI Symposium on Engineering Interactive Computing Systems, EICS '17, pp. 33-38, ACM, New York, NY, USA, https://doi.org/10.1145/3102113.3102122, 2017.

Weakliam, J., Bertolotto, M., and Wilson, D.: Implicit Interaction Profiling for Recommending Spatial Content, in: Proceedings of the 13th Annual ACM International Workshop on Geographic Information Systems, GIS '05, pp. 285-294, ACM, New York, NY, USA, https://doi.org/10.1145/1097064.1097104, 2005. 